OAI-PMH: http://www.indteca.com/ojs/index.php/Revista Scientific/oai

Artículo Original / Original Article

\title{
Los Estudiantes de Pregrado en Ecuador: Un Análisis de Datos
}

\author{
Autor: Efstathios Stefos \\ Universidad Nacional de Educación, UNAE \\ Proyecto INÉDITA, SENESCYT \\ stefos.efstathios@unaeedu.onmicrosoft.com \\ Azogues, Ecuador \\ https://orcid.org/0000-0002-5679-8002
}

\section{Resumen}

El objetivo del estudio es analizar y presentar características demográficas y sociales de los estudiantes de pregrado en Ecuador, para lo cual se ha realizado un análisis estadístico descriptivo y un análisis estadístico multidimensional utilizando datos de la Encuesta Nacional de Empleo, Desempleo y Subempleo del año 2017. El 86.26\% de los estudiantes matriculados en Universidades del Ecuador vive en área urbana y el $13.74 \%$ en área rural; el $89.39 \%$ se consideran mestizos, el $3.35 \%$ indígenas, el $4.25 \%$ afroecuatorianos, negros o mulatos, el $1.67 \%$ se consideran montubios y el $1.29 \%$ blancos; el $66.60 \%$ de los alumnos de pregrado en Ecuador estudia en universidades públicas y el $32.54 \%$ en universidades particulares; el $8.27 \%$ se consideran pobres.

Palabras clave: estudiante; Ecuador; análisis de datos.

Cómo citar este artículo:

Stefos, E. (2019). Los Estudiantes de Pregrado en Ecuador: Un Análisis de Datos. Revista Scientific, 4(14), 85-100, e-ISSN: 2542-2987. Recuperado de: https://doi.org/10.29394/Scientific.issn.25422987.2019.4.14.4.85-100

Fecha de Recepción: 13-07-2019
Fecha de Aceptación:

19-09-2019
Fecha de Publicación:

05-11-2019 


\title{
Undergraduate Students in Ecuador: A Data Analysis
}

\begin{abstract}
The objective of the study is to analyze and present demographic and social characteristics of undergraduate students in Ecuador, for which a descriptive statistical analysis and a multidimensional statistical analysis have been performed using data from the National Survey of Employment, Unemployment and Under-employment of 2017. $86.26 \%$ of the students enrolled in Universities of Ecuador live in urban areas and $13.74 \%$ in rural areas; $89.39 \%$ are considered mestizos, 3.35\% indigenous, 4.25\% AfroEcuadorians, blacks or mulattos, $1.67 \%$ are considered montubios and $1.29 \%$ are white; $66.60 \%$ of undergraduate students in Ecuador study in public universities and $32.54 \%$ in private universities; $8.27 \%$ consider themselves poor.
\end{abstract}

Keywords: student; Ecuador; data analysis.

How to cite this article:

Stefos, E. (2019). Undergraduate Students in Ecuador: A Data Analysis. Revista Scientific, 4(14), 85100, e-ISSN: 2542-2987. Recovered from: https://doi.org/10.29394/Scientific.issn.2542$\underline{2987.2019 .4 .14 .4 .85-100}$

Date Received:

13-07-2019
Date Acceptance:

19-09-2019
Date Publication:

05-11-2019 


\section{Introducción}

El objetivo del presente estudio es determinar, a través de un análisis estadístico, y presentar características demográficas y sociales de los estudiantes de pregrado en Ecuador.

De acuerdo con el Reglamento General a la Ley Orgánica de Educación Intercultural (LOEI) del Ministerio de Educación (2012a), el nivel de Educación General Básica (EGB) se divide en 4 subniveles (de 5 a 14 años de edad):

1. Preparatoria, que corresponde a $1 . .9$ grado de Educación General Básica y preferentemente se ofrece a los estudiantes de cinco (5) años de edad; 2. Básica Elemental, que corresponde a $2 .$. , $3 .$. y $4 . .9$ grados de Educación General Básica y preferentemente se ofrece a los estudiantes de 6 a 8 años de edad; 3. Básica Media, que corresponde a 5.․, 6․․ y 7.ำ grados de Educación General Básica y preferentemente se ofrece a los estudiantes de 9 a 11 años de edad; y, 4. Básica Superior, que corresponde a 8.․ 9.. y 10.ำ grados de Educación General Básica y preferentemente se ofrece a los estudiantes de 12 a 14 años de edad (pág. 9).

De acuerdo con el Reglamento General a la Ley Orgánica de Educación Intercultural del Ministerio de Educación (2012b): después de la EGB sigue el Bachillerato que tiene 3 niveles y principalmente es ofertado a los estudiantes de 15 a 17 años de edad (pág. 9).

Según López y Tedesco (2002): posteriormente del Bachillerato, siguen los estudios de pregrado que incluyen alumnos de 18 años de edad o más (pág. 56).

El presente estudio analiza y presenta las características demográficas y sociales de los estudiantes de pregrado en Ecuador y los resultados del estudio pueden utilizarse como una herramienta para la formulación de políticas públicas relacionadas con el Sistema Nacional de Educación del país. 


\section{Metodología}

El Instituto Nacional de Estadística y Censos (INEC, 2017a): ofreció los datos de la Encuesta Nacional de Empleo, Desempleo y Subempleo que se utilizaron en el presente estudio (pág. 1). En este estudio se realizó: un análisis descriptivo, en el cual se aplican frecuencias y porcentajes; y además un análisis con estadística multidimensional.

De acuerdo con Athanasiadis (1995): el análisis multidimensional se utiliza con el fin de mostrar, principalmente, criterios de diferenciación y la clasificación en grupos más importantes de las personas investigadas (pág. 53). Los métodos utilizados son: el análisis factorial de correspondencias múltiples para analizar los criterios de diferenciación, y la agrupación jerárquica que propone y además determina grupos de personas por sus características comunes.

Según Morineau (1984): el análisis factorial de correspondencias múltiples se usa para determinar cómo las personas se diferencian según las respuestas dadas a la encuesta analizada. Es decir que el método investiga y da como resultado la correlación de las variables de la encuesta de manera simultánea (pág. 23). Como recomiendan Costa, Beuren, Sarquis y Sampaio (2015): se analizaron indicadores sociales y se definieron los ejes factoriales como los criterios que diferencian a los encuestados y expresan las oposiciones en sus respuestas (pág. 81).

De acuerdo con Olivier (2008): el análisis jerárquico, en cambio agrupa a las personas encuestadas y muestra estos grupos de acuerdo a sus respuestas o características comunes (pág. 86). Este método también presenta un gráfico de clasificación que conecta estos grupos.

\section{Resultados del análisis descriptivo}

Según los datos de la Encuesta Nacional de Empleo y Subempleo del Instituto Nacional de Estadística y Censos (INEC, 2017b): en las siguientes 
tablas se presenta el perfil social de los estudiantes matriculados en las Universidades del Ecuador (pág. 1).

Tabla 1. Área.

\begin{tabular}{|l|r|r|}
\cline { 2 - 3 } \multicolumn{1}{c|}{} & \multicolumn{1}{c|}{$\mathrm{n}$} & \multicolumn{1}{c|}{$\%$} \\
\hline Urbana & 472333 & 86.26 \\
\hline Rural & 75225 & 13.74 \\
\hline Total & 547558 & 100.00 \\
\hline
\end{tabular}

Fuente: El Autor (2017); Elaboración a partir de la ENEMDU.

El $86.26 \%$ de los estudiantes matriculados en Universidades del Ecuador vive en área urbana y el $13.74 \%$ en área rural, expuesto en la tabla 1.

Tabla 2. Sexo.

\begin{tabular}{|l|r|r|}
\cline { 2 - 3 } \multicolumn{1}{c|}{} & \multicolumn{1}{c|}{$\mathrm{n}$} & \multicolumn{1}{c|}{$\%$} \\
\hline Hombre & 263527 & 48.13 \\
\hline Mujer & 284031 & 51.87 \\
\hline Total & 547558 & 100.00 \\
\hline
\end{tabular}

Fuente: El Autor (2017); Elaboración a partir de la ENEMDU.

El $48.13 \%$ de los estudiantes son hombres y el $51.87 \%$ mujeres, como se observa en la tabla 2.

Tabla 3. Edad.

\begin{tabular}{|l|r|r|}
\cline { 2 - 3 } \multicolumn{1}{c|}{} & \multicolumn{1}{c|}{$\mathrm{n}$} & \multicolumn{1}{c|}{$\%$} \\
\hline $18-22$ & 334764 & 61.14 \\
\hline $23-29$ & 163222 & 29.81 \\
\hline Igual o mayor que 30 & 49573 & 9.05 \\
\hline Total & 547558 & 100.00 \\
\hline
\end{tabular}

Fuente: El Autor (2017); Elaboración a partir de la ENEMDU. 
El $61.14 \%$ de los estudiantes del pregrado tiene de 18 a 22 años de edad, el $29.81 \%$ tiene de 23 a 29 años y el $9.05 \%$ es igual o mayor que 30 años de edad, presentado en la tabla 3.

Tabla 4. Estado civil.

\begin{tabular}{|l|r|r|}
\cline { 2 - 3 } \multicolumn{1}{c|}{} & \multicolumn{1}{c|}{$\mathrm{n}$} & \multicolumn{1}{c|}{$\%$} \\
\hline Casado(a) & 40309 & 7.36 \\
\hline Separado(a) & 11384 & 2.08 \\
\hline Divorciado(a) & 3020 & 0.55 \\
\hline Viudo(a) & 411 & 0.08 \\
\hline Unión libre & 37635 & 6.87 \\
\hline Soltero(a) & 454800 & 83.06 \\
\hline Total & 547558 & 100.00 \\
\hline
\end{tabular}

Fuente: El Autor (2017); Elaboración a partir de la ENEMDU.

El $83.06 \%$ de los estudiantes de pregrado en Ecuador son solteros, el $14.23 \%$ casados o en unión libre, el $2.63 \%$ separados o divorciados y el $0.08 \%$ son viudos, mostrado en la tabla 4.

Tabla 5. Asistencia a clases.

\begin{tabular}{|l|r|r|}
\cline { 2 - 3 } \multicolumn{1}{c|}{} & \multicolumn{1}{c|}{$\mathrm{n}$} & \multicolumn{1}{c|}{$\%$} \\
\hline Si & 545398 & 99.61 \\
\hline No & 2160 & 0.39 \\
\hline Total & 547558 & 100.00 \\
\hline
\end{tabular}

Fuente: El Autor (2017); Elaboración a partir de la ENEMDU.

Únicamente el $0.39 \%$ de los estudiantes de pregrado en Ecuador no asiste a clases, indicado en la tabla 5.

Tabla 6. Idioma.

\begin{tabular}{|l|r|r|}
\cline { 2 - 3 } \multicolumn{1}{c|}{} & \multicolumn{1}{c|}{$\mathrm{n}$} & \multicolumn{1}{c|}{$\%$} \\
\hline Solo lengua indígena & 152 & 0.03 \\
\hline Lengua indígena y español & 11102 & 2.03 \\
\hline
\end{tabular}




\begin{tabular}{|l|r|r|}
\hline Solo español & 502438 & 91.76 \\
\hline Español e idioma extranjero & 32461 & 5.93 \\
\hline Lengua indígena e idioma extranjero & 992 & 0.18 \\
\hline Idioma extranjero & 413 & 0.08 \\
\hline Total & 547558 & 100.00 \\
\hline
\end{tabular}

Fuente: El Autor (2017); Elaboración a partir de la ENEMDU.

El $91.76 \%$ de los estudiantes habla solo español, el 5.93\% habla español e idioma extranjero y el $0.03 \%$ habla solo lengua indígena, como se muestra en la tabla 6 .

Tabla 7. Autoidentificación étnica.

\begin{tabular}{|l|r|r|}
\cline { 2 - 3 } \multicolumn{1}{c|}{} & \multicolumn{1}{c|}{$\mathrm{n}$} & \multicolumn{1}{c|}{$\%$} \\
\hline Indígena & 18360 & 3.35 \\
\hline Afroecuatoriano & 8674 & 1.58 \\
\hline Negro & 7749 & 1.42 \\
\hline Mulato & 6840 & 1.25 \\
\hline Montubio & 9152 & 1.67 \\
\hline Mestizo & 489456 & 89.39 \\
\hline Blanco & 7041 & 1.29 \\
\hline Otro & 288 & 0.05 \\
\hline Total & 547558 & 100.00 \\
\hline
\end{tabular}

Fuente: El Autor (2017); Elaboración a partir de la ENEMDU.

El $89.39 \%$ de los estudiantes de pregrado se consideran mestizos, el $3.35 \%$ indígenas, el $4.25 \%$ afroecuatorianos, negros o mulatos, el $1.67 \%$ se consideran montubios y el $1.29 \%$ blancos, como se exhibe en la tabla 7 .

Tabla 8. Estudiantes matriculados por edad y año de estudios.

\begin{tabular}{|l|r|}
\cline { 2 - 2 } \multicolumn{1}{c|}{} & $\mathrm{n}$ \\
\hline 18 años / primer año & 60112 \\
\hline 19 años / segundo año & 21215 \\
\hline 20 años / tercer año & 15797 \\
\hline 21 años / cuarto año & 11897 \\
\hline 22 / 5to año & 7630 \\
\hline
\end{tabular}

Fuente: El Autor (2017); Elaboración a partir de la ENEMDU. 
Los estudiantes correspondientes al primer año de estudios que tienen 18 años de edad son 60112, de segundo año que tienen 19 años de edad son 21215, de tercer año que tienen 20 años de edad son 15797, de cuarto año que tienen 21 años de edad son 11897 y de quinto año que tienen 22 años de edad son 7630 , como se indica en la en la tabla 8.

Tabla 9. El establecimiento donde se matriculó es:

\begin{tabular}{|l|r|r|}
\cline { 2 - 3 } \multicolumn{1}{c|}{} & \multicolumn{1}{c|}{$\mathrm{n}$} & \multicolumn{1}{c|}{$\%$} \\
\hline Fiscal & 364696 & 66.60 \\
\hline Particular & 178415 & 32.58 \\
\hline Municipal, fiscomisional & 4447 & 0.81 \\
\hline Total & 547558 & 100.00 \\
\hline
\end{tabular}

Fuente: El Autor (2017); Elaboración a partir de la ENEMDU.

El $66.60 \%$ de los estudiantes de pregrado en Ecuador estudia en universidades públicas y el $32.58 \%$ en universidades particulares, como se presenta en la tabla 9.

Tabla 10. Rama de actividad.

\begin{tabular}{|l|r|r|}
\cline { 2 - 3 } \multicolumn{1}{l|}{} & $\mathrm{n}$ & $\%$ \\
\hline Agricultura, ganadería, caza, silvicultura y pesca & 11687 & 2.13 \\
\hline Explotación de minas y canteras & 340 & 0.06 \\
\hline Industrias manufactureras & 18379 & 3.36 \\
\hline Suministros de electricidad, gas, aire acondicionado & 1322 & 0.24 \\
\hline Distribución de agua, alcantarillado & 436 & 0.08 \\
\hline Construcción & 4471 & 0.82 \\
\hline Comercio, reparación de vehículos & 45948 & 8.39 \\
\hline Transporte y almacenamiento & 5880 & 1.07 \\
\hline Actividades de alojamiento y servicios de comida & 15007 & 2.74 \\
\hline Información y comunicación & 5060 & 0.92 \\
\hline Actividades financieras y de seguros & 4086 & 0.75 \\
\hline Actividades inmobiliarias & 504 & 0.09 \\
\hline Actividades profesionales, científicas y técnicas & 8412 & 1.54 \\
\hline Actividades y servicios administrativos y de apoyo & 5988 & 1.09 \\
\hline Administración pública, defensa y seguridad social & 7787 & 1.42 \\
\hline
\end{tabular}




\begin{tabular}{|l|r|r|} 
Enseñanza & 13469 & 2.46 \\
\hline Actividades, servicios sociales y de salud & 8707 & 1.59 \\
\hline Artes, entretenimiento y recreación & 2326 & 0.42 \\
\hline Otras actividades de servicios & 4705 & 0.86 \\
\hline Actividades en hogares privados con servicio doméstico & 568 & 0.10 \\
\hline NA & 382479 & 69.85 \\
\hline Total & 547558 & 100.00 \\
\hline
\end{tabular}

Fuente: El Autor (2017); Elaboración a partir de la ENEMDU.

De los estudiantes de pregrado, 45948 trabajan en el comercio o reparación de vehículos, 18379 en industrias manufactureras, 15007 en actividades de alojamiento y servicios de comida, 13469 en actividades de enseñanza, 11687 en agricultura, ganadería, caza, silvicultura y pesca, y 60592 alumnos en otras actividades, como se expone en la tabla 10.

Tabla 11. Pobreza.

\begin{tabular}{|l|r|r|}
\cline { 2 - 3 } \multicolumn{1}{c|}{} & \multicolumn{1}{c|}{$\mathrm{n}$} & \multicolumn{1}{c|}{$\%$} \\
\hline Pobre & 45259 & 8.27 \\
\hline No pobre & 497773 & 90.91 \\
\hline NA & 4526 & 0.83 \\
\hline Total & 547558 & 100.00 \\
\hline
\end{tabular}

Fuente: El Autor (2017); Elaboración a partir de la ENEMDU.

El $8.27 \%$ de los estudiantes de pregrado en Ecuador se consideran pobres, mostrado en la tabla 11.

Tabla 12. Extrema pobreza.

\begin{tabular}{|l|r|r|}
\cline { 2 - 3 } \multicolumn{1}{c|}{} & \multicolumn{1}{c|}{$\mathrm{n}$} & \multicolumn{1}{c|}{$\%$} \\
\hline Indigente & 9968 & 1.82 \\
\hline No indigente & 533064 & 97.35 \\
\hline NA & 4526 & 0.83 \\
\hline Total & 547558 & 100.00 \\
\hline
\end{tabular}

Fuente: El Autor (2017); Elaboración a partir de la ENEMDU. 
El $1.82 \%$ de los estudiantes de pregrado se consideran indigentes, como se visualiza en la tabla 12 .

\section{Resultados del análisis factorial de correspondencias múltiples}

El análisis factorial de correspondencias múltiples se usó para poder determinar cómo los ecuatorianos que asisten a clases de pregrado son diferenciados según las respuestas dadas en la encuesta usada para este estudio. Como resultado, los criterios que diferencian a los encuestados son los siguientes:

Primer criterio de diferenciación (inercia 12.13\%): En el primer eje, por un lado, hay estudiantes que se consideran pobres e indigentes, tienen de 18 a 22 años de edad, viven en área rural, se consideran indígenas, hablan lengua indígena y español, se encuentran cursando el primer año de estudios en una universidad pública y son solteros. Por otro lado, hay estudiantes que no se consideran pobres, viven en área urbana, se consideran mestizos, estudian en el $4^{\circ} \circ 5^{\circ}$ año en una universidad particular, tienen 23 y más años de edad, y son casados o en unión libre.

Segundo criterio de diferenciación (inercia 10.64\%): En el segundo eje, por un lado, hay estudiantes solteros de 18 a 22 años de edad que se consideran mestizos y hablan solo español, no se consideran pobres, se encuentran en el primer o segundo año de sus estudios en una universidad pública, son hombres y viven en área urbana. Por otro lado, hay estudiantes que se consideran indígenas, hablan lengua indígena y español, tienen 23 y más años de edad, son casados o en unión libre, y están cursando el 4ํㅜ y $5^{\circ}$ año de sus estudios en una universidad particular.

Tercer criterio de diferenciación (inercia 8.19\%): En el tercer eje, por un lado, hay estudiantes que tienen 23 y más años de edad, se encuentran en el $4^{\circ}$ y $5^{\circ}$ año de sus estudios en una universidad pública, se consideran pobres e indigentes, son solteros o en unión libre, y se consideran mestizos. Por otro 
lado, hay estudiantes de 18 a 22 años de edad que no se consideran pobres y se consideran indígenas.

\section{Resultados del análisis jerárquico}

El análisis jerárquico determinó cinco grupos de 547558 personas que se presentan en la Figura 1.

Figura 1. El gráfico de clasificación.

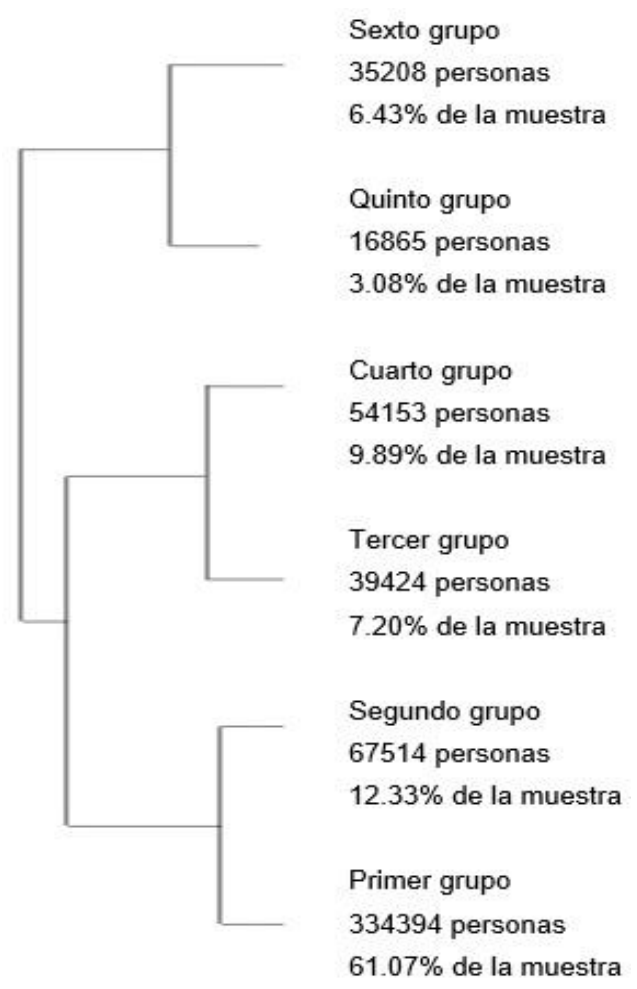


Segundo grupo (67514 personas, $12.33 \%$ de la muestra): Los estudiantes del segundo grupo están cursando el quinto año de sus estudios en una universidad particular, hablan español e idioma extranjero, tienen de 23 a 29 años de edad, son solteros, y no se consideran pobres.

Tercer grupo (39424 personas, $7.20 \%$ de la muestra): Los estudiantes del tercer grupo tienen 23 y más años de edad, están en unión libre, son mujeres, están cursando el tercer año de sus estudios, hablan solo español, no se consideran pobres, y viven en área urbana.

Cuarto grupo (54153 personas, 9.89\% de la muestra): Los estudiantes del cuarto grupo están estudiando en una universidad particular, la mayoría de ellos están casados, tienen 30 y más años de edad, no se consideran pobres, están cursando el cuarto año de sus estudios, y se consideran mestizos.

Quinto grupo (16865 personas, 3.08\% de la muestra): Los estudiantes del quinto grupo se consideran pobres e indigentes, viven en área rural, la mayoría de ellos se consideran indígenas, están estudiando en una universidad pública, y hablan lengua indígena y español.

Sexto grupo (35208 personas, $6.43 \%$ de la muestra): Los estudiantes del sexto grupo se consideran mestizos e indígenas, hablan lengua indígena y español, viven en área rural, se consideran pobres, y están cursando el primero y el quinto año de sus estudios.

Las diferencias de los grupos se muestran en la figura 2, en donde el gráfico del Análisis de Correspondencias (nivel factorial 1×2) presenta los centroides de los 6 grupos en dos ejes. También, determina las diferencias y las similitudes entre las personas de los seis grupos. 
Figura 2. Análisis de correspondencias.

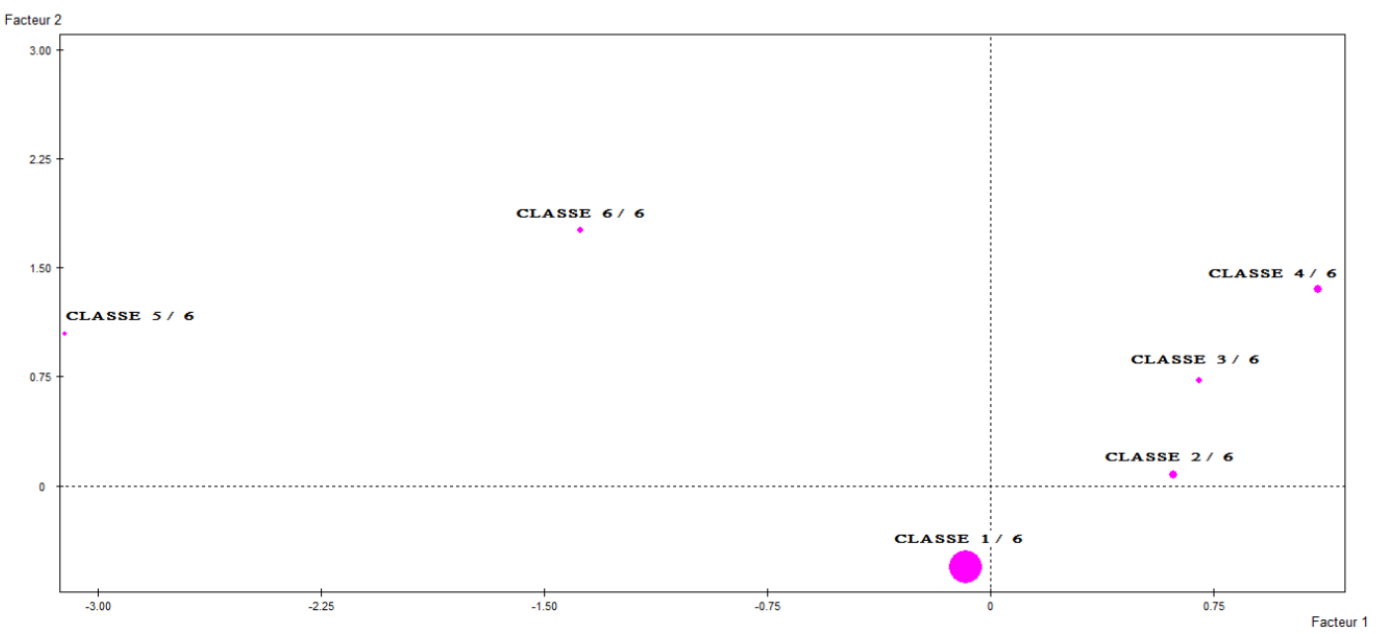

Fuente: El Autor (2017); Elaboración a partir de la ENEMDU.

\section{Conclusiones}

En este estudio se analizó y determinó el perfil de los estudiantes de pregrado en Ecuador realizando un análisis estadístico descriptivo y un análisis estadístico multidimensional con el objetivo de mostrar las frecuencias y los porcentajes de las variables y los principales criterios que diferencian y clasifican en grupos las personas investigadas. Los métodos empleados en este estudio fueron dos: el análisis factorial de correspondencias múltiples y el análisis jerárquico.

Los principales resultados que se obtuvieron en el estudio descriptivo y fueron confirmados por el análisis factorial son los siguientes:

De acuerdo con el análisis que se realizó el $86.26 \%$ de los estudiantes matriculados en las Universidades del Ecuador vive en área urbana y el $13.74 \%$ en área rural; el $48.13 \%$ son hombres y el $51.87 \%$ mujeres.

El $61.14 \%$ de los estudiantes de pregrado tiene de 18 a 22 años de edad, el $29.81 \%$ tiene de 23 a 29 años y el $9.05 \%$ es igual o mayor que 30 
años de edad. El $83.06 \%$ son solteros, el $14.23 \%$ casados o en unión libre, el $2.63 \%$ separados o divorciados y el $0.08 \%$ son viudos. El $99.61 \%$ de los estudiantes asiste a clases.

El $91.76 \%$ habla solo español, el 5.93\% habla español e idioma extranjero y el $0.03 \%$ habla solo lengua indígena. El $89.39 \%$ se consideran mestizos, el $3.35 \%$ indígenas, el $4.25 \%$ afroecuatorianos, negros o mulatos, el $1.67 \%$ se consideran montubios y el $1.29 \%$ blancos.

Los estudiantes del primer año de estudios que tienen 18 años de edad son 60112, del segundo año que tienen 19 años de edad son 21215, del tercer año que tienen 20 años de edad son 15797, del cuarto año que tienen 21 años de edad son 11897 y den quinto año que tienen 22 años de edad son 7630 . El $66.60 \%$ de los alumnos de pregrado en Ecuador estudia en universidades públicas y el $32.54 \%$ en universidades particulares.

45948 de los estudiantes del pregrado trabajan en el comercio o reparación de vehículos, 18379 en industrias manufactureras, 15007 en actividades de alojamiento y servicios de comida, 13469 en actividades de enseñanza, 11687 en agricultura, ganadería, caza, silvicultura y pesca, y 60592 alumnos en otras actividades. El $8.27 \%$ se consideran pobres y el $1.82 \%$ indigentes.

\section{Referencias}

Athanasiadis, I. (1995). Correspondence Analysis and Hierarchical Classification. Athens, Greece: New Technologies Editions.

Costa, P., Beuren, I., Sarquis, R., \& Sampaio, G. (2015). Análisis de la homogeneidad de indicadores sociales de los países de América Latina y el Caribe. GCG. Revista de Globalización, Competitividad y Gobernabilidad, 9(1), 74-96, e-ISSN: 1988-7116. Recuperado de: https://doi.org/10.3232/GCG.2015.V9.N1.04 INEC (2017a,b). Encuesta Nacional de Empleo, Desempleo y Subempleo 
ENEMDU. Quito, Ecuador: Instituto Nacional de Estadística y Censos.

Recuperado de: https://www.ecuadorencifras.gob.ec/enemdu-2017/

López, N., \& Tedesco, J. (2002). Challenges for Secondary Education in

Latin America. CEPAL Review 76, 55-68. Santiago de Chile: CEPAL -

Naciones Unidas. Recuperado de:

http://repositorio.cepal.org/bitstream/handle/11362/10862/1/76055068I

\begin{abstract}
en.pdf
Ministerio de Educación (2012a,b). Reglamento General a la Ley Orgánica de Educación Intercultural. Quito, Ecuador: Dado en el Palacio Nacional por la Dirección Nacional de Normativa Jurídico Educativa del Ministerio de Educación. Recuperado de: https://educacion.gob.ec/wpcontent/uploads/downloads/2017/02/Reglamento-General-a-la-Ley-

OrgAnica-de-Educacion-Intercultural.pdf

Morineau, A. (1984). Note sur la Caracterisation Statistique d'une Classe et les Valeurs-tests. Bulletin Technique du Centre de Statistique et d'Informatique Appliquées, 2(1-2), 20-27. Francia: Association CESIA. Recuperado de: http://www.deenov.com/Data/Sites/1/docs/ValeurTest-critere-de-caracterisation-statistique.pdf
\end{abstract}

Olivier, M. (2008). The analysis of quantitative data. Athens, Greece: Topos. 


\section{Efstathios Stefos}

e-mail: stefos.efstathios@unae.edu.ec

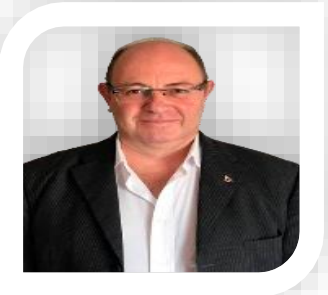

Nacido en Volos, Grecia, el 12 de noviembre del año 1967. Profesor Principal Titular, Vicerrector Académico y miembro de la Comisión Gestora de la Universidad Nacional de Educación (UNAE); tiene $\mathrm{PhD}$. en Estudios Pedagógicos; Licenciatura en la Educación; Especialización en Dificultades de Aprendizaje; Maestría en Diseño Ambiental; y Diploma en Ingeniería Eléctrica. Tiene dos vinculaciones con el Proyecto Prometeo, una en el proyecto "El desarrollo de la función de producción educativa ecuatoriana a través de las redes neuronales artificiales" y otra en el proyecto "Desarrollo de capacidades en Biometría". Ha publicado los resultados de sus investigaciones en revistas científicas indexadas de impacto mundial, libros, capítulos de libros y congresos. Tiene experiencia educativa internacional y experiencia significativa en la gestión como docente e investigador de universidades y centros de investigación de prestigio; Director de Evaluación; editor asociado y miembro de comités editoriales de revistas reconocidas internacionalmente; miembro de consejos regionales de educación; delegado a la Asamblea Nacional para reformas de las leyes orgánicas de educación en Ecuador, etc. 